\title{
Legacy publisher
}

Public Domain

\section{Source}

Open Research Glossary.

A publisher that historically has operated on a paywall-based business model. 